\title{
Knockdown of TWIST enhances the cytotoxicity of chemotherapeutic drugs in doxorubicin-resistant HepG2 cells by suppressing MDR1 and EMT
}

\author{
RONG LI ${ }^{1}$, CHANGLI WU ${ }^{2}$, HONGYING LIANG ${ }^{3}$, YINGHAI ZHAO ${ }^{1}$, \\ CHUNYAN LIN $^{3}$, XIUJUAN ZHANG ${ }^{2}$ and CAIGUO YE ${ }^{4}$ \\ Departments of ${ }^{1}$ Pathology and Pathophysiology and ${ }^{2}$ Physiology, ${ }^{3}$ Laboratory of Physiological Science, \\ Guangdong Medical University, Zhanjiang, Guangdong 524023; ${ }^{4}$ China-America United Cancer Research Institute, \\ Guangdong Medical University, Dongguan, Guangdong 523800, P.R. China
}

Received January 31, 2018; Accepted May 31, 2018

DOI: 10.3892/ijo.2018.4495

\begin{abstract}
The transcription factor twist family bHLH transcription factor 1 (TWIST), which is a member of the basic helix-loop-helix class of proteins, is known to induce epithelial-mesenchymal transition (EMT) and promote cancer metastasis. TWIST has previously been reported to be associated with multidrug resistance (MDR), since its depletion increases drug sensitivity. Although these previous studies have established a strong association between EMT and MDR, the molecular mechanism remains obscure. The present study demonstrated that TWIST protein expression was elevated in liver cancer, and was positively correlated with multidrug resistance protein 1 (MDR1) expression. Conversely, MDR1 was negatively correlated with E-cadherin expression in liver cancer samples. In addition, the present study indicated that doxorubicin-resistant HepG2 (R-HepG2) cells acquired an EMT phenotype. TWIST was also more highly expressed in R-HepG2 cells compared with in parental HepG2 cells. Knockdown of TWIST increased the sensitivity of R-HepG2 cells to 5-fluroracil, cisplatin and doxorubicin through a reduction in MDR1 expression and drug efflux ability. Furthermore, knockdown of TWIST in R-HepG2 cells inhibited the migratory ability of cells and suppressed the EMT phenotype. These findings demonstrated that targeting TWIST may be considered a novel strategy to overcome drug resistance in liver cancer.
\end{abstract}

Correspondence to: Dr Xiujuan Zhang, Department of Physiology, Guangdong Medical University, 2 East Wenming Road, Xiashan, Zhanjiang, Guangdong 524023, P.R. China

E-mail: luciazxj@163.com

Dr Caiguo Ye, China-America United Cancer Research Institute, Guangdong Medical University, 1 XinCheng Road, Songshan Lake, Dongguan, Guangdong 523800, P.R. China

E-mail: yecaiguo@gdmu.edu.cn

Key words: liver cancer, multidrug resistance, epithelial-mesenchymal transition, TWIST

\section{Introduction}

Liver cancer is one of the most common types of malignant tumor worldwide, which is associated with relatively poor prognosis and rapid progression $(1,2)$. Liver cancer consists of two subtypes: Hepatocellular carcinoma and hepatoblastoma. It has previously been reported that $20 \%$ of patients with unresectable liver cancer exhibit reduced sensitivity to chemotherapy, and no survival benefit is observed. Although chemotherapy prolongs patient survival, a large number of patients with cancer benefit less due to a poor response to chemotherapeutic drugs $(3,4)$. Multidrug resistance (MDR) serves crucial roles in drug-resistant cancer cells (5). Multidrug resistance protein 1 (MDR1) serves as an ATP-dependent drug efflux pump that reduces the intracellular accumulation of chemotherapeutic drugs, such as cisplatin (DDP), 5-fluoracil (5-FU) and doxorubicin, and thereby limits their anticancer efficacy $(6,7)$. Due to the important role of MDR1 in drug resistance, blockade of MDR1 may help to avert drug resistance.

Twist family bHLH transcription factor 1 (TWIST) is a highly conserved basic helix-loop-helix transcription factor, which has an important role in epithelial-mesenchymal transition (EMT). EMT is a transient phase initially observed in embryonic development $(8,9)$. Downregulation of TWIST by small interfering RNA leads to decreased metastatic potential and invasion of prostate carcinoma cells $(10,11)$.

Recent studies have reported that EMT is associated with chemoresistance in cancer $(12,13)$. Consistent with a previous report (14), it was revealed that TWIST is involved in the development of acquired drug resistance in human cancer cells. TWIST overexpression is also correlated with chemotherapy resistance in various types of cancer and leads to a poorer prognosis $(15,16)$. Therefore, TWIST may be considered a novel therapeutic target in overcoming MDR in liver cancer. The present study aimed to investigate the relationship between TWIST and MDR1 in liver cancer cell-associated drug resistance.

The present study provided evidence to suggest that TWIST was highly expressed in liver cancer tissues and was positively correlated with MDR1 expression. Furthermore, 
the results confirmed that MDR1 was negatively correlated with E-cadherin expression in cancer samples. Knockdown of TWIST enhanced the cytotoxicity of chemotherapeutic drugs in R-HepG2 cells by suppressing MDR1 and reversing EMT.

\section{Materials and methods}

Tissue specimens. A total of 49 paraffin-embedded samples were obtained from patients who were diagnosed at the Affiliated Hospital of the Guangdong Medical University (Zhanjiang, China) between April and September 2015. Patient characteristics are presented in Table I. A total of 22 corresponding non-cancerous liver tissues were also obtained immediately following surgical resection; these 22 non-cancerous tissues were paired with cancer samples. However, not all corresponding non-cancerous samples were collected for all cancer samples as some non-cancerous samples were missing due to surgical reasons. All patients signed a consent form, which disclosed that the samples were to be used for scientific research. None of the patients received preoperative therapy, such as with transarterial chemoembolization or percutaneous ethanol injection. The present study was approved by the Institutional Ethics Committee of Guangdong Medical University. All patients provided written informed consent.

Immunohistochemistry (IHC). Paraffin-embedded sections $(4 \mu \mathrm{m})$ were prepared for IHC. Briefly, sections were blocked with $2 \%$ bovine serum albumin (cat. no. 05470; Sigma-Aldrich; Merck KGaA, Darmstadt, Germany) at room temperature for $1 \mathrm{~h}$, followed by the clearance of endogenous peroxidases with $0.3 \% \mathrm{H}_{2} \mathrm{O}_{2}$ for $15 \mathrm{~min}$. Sections were then incubated with primary antibodies against TWIST (Abcam, Cambridge, MA, USA, cat. no. ab49254), MDR1 (cat. no. ab170904; Abcam), E-cadherin (cat. no. sc-7870; Santa Cruz Biotechnology, Inc., Dallas, TX, USA) or vimentin (cat. no. 3932; Cell Signaling Technology Inc., Danvers, MA, USA) at $4^{\circ} \mathrm{C}$ overnight, whereas non-immune immunoglobulin $\mathrm{G}$ (IgG, cat. no. A7016; Beyotime Institute of Biotechnology, Haimen, China) was used as a negative control. Antigenic sites were localized using a SP9000 kit and DAB kit (cat. nos. SP9000 and C02-100; OriGene Technologies, Inc., Beijing, China). Horseradish peroxidase-labeled secondary antibodies were subsequently added to the sections at room temperature for $1 \mathrm{~h}$ according to the manufacturer's protocol (supplied with the DAB kit, cat. no. C02-100; Origene Technologies, Inc.). IHC staining results were interpreted independently by two pathologists (DM300 LED; Leica Microsystems GmbH, Wetzlar, Germany). Briefly, the immunoreactive scores (IRS) of TWIST, MDR1, E-cadherin and vimentin were calculated as follows: 0, negative; 1 , weak; 2 , moderate and 3 , strong. The percentage of positive cells was scored as $1,0-9 \% ; 2,10-50 \% ; 3,51-75 \%$ and $4,>76 \%$. The two scores were multiplied together and samples with a total IRS of 0-1, 2-3, 4-5 and $\geq 6$ were considered (-), (+), $(++)$ and $(+++)$, respectively. According to the final score, the expression levels of TWIST, MDR1, E-cadherin and Vimentin were categorized as low $(<6)$ or high $(\geq 6)$.

Cell cultures and establishment of a TWIST-silenced cell line. HepG2 cells (cat. no. HB-8065; American Type Culture
Collection, Manassas, VA, USA) were cultured in RPMI-1640 medium (Gibco; Thermo Fisher Scientific, Inc., Waltham, MA, USA) supplemented with $10 \%$ (v/v) fetal bovine serum (FBS; Gibco; Thermo Fisher Scientific, Inc.), $100 \mathrm{U} / \mathrm{ml}$ penicillin G and $100 \mu \mathrm{g} / \mathrm{ml}$ streptomycin. HepG2 cells were initially misidentified as a hepatocellular carcinoma cell line; however, they have now been identified as hepatoblastoma cells (17). Cells were incubated at $37^{\circ} \mathrm{C}$ in a humidified atmosphere containing $5 \% \mathrm{CO}_{2}$. To maintain drug resistance, doxorubicinresistant HepG2 (R-HepG2) cells were cultured for 2 weeks and continuously maintained in medium containing $1.2 \mu \mathrm{M}$ doxorubicin (cat. no. D1515; Sigma-Aldrich; Merck KGaA). R-HepG2 cells were provided by Dr Ye Caiguo (Chinese University of Hong Kong, Hong Kong, China). Doxorubicin resistance was induced by continued incubation of the parental HepG2 cells with $1.2 \mu \mathrm{M}$ doxorubicin, until a single stable clone was obtained, which was named R-HepG2.

TWIST-silenced HepG2 and R-HepG2, and negative control (NC) HepG2 and R-HepG2 cell lines were established as previously described (18). Packaged lentiviruses containing TWIST-specific short hairpin RNA (shRNA), which were labeled with green fluorescent protein as a transfection marker were provided by Shanghai GenePharma Co., Ltd. (Shanghai, China). The shRNA sequences were as follows: TWIST shRNA, 5'-CCTGA GCAACAGCGAGGAA-3'; and NC shRNA, 5'-TTCTCCGA ACGTGTCACGT-3'. Cells were infected with lentiviruses (multiplicity of infection=25) for $24 \mathrm{~h}$ at $37^{\circ} \mathrm{C}$. Subsequently, cells were cultured with fresh complete RPMI- 1640 medium containing $1 \mu \mathrm{g} / \mathrm{ml}$ puromycin for 14 days. The efficiency of TWIST silencing was determined by western blotting. Stable TWIST-silenced HepG2 or R-HepG2 cells (si-TWIST group), and NC HepG2 or R-HepG2 cells (NC group) were maintained in similar medium to HepG2 or R-HepG2 cells supplemented with $0.5 \mu \mathrm{g} / \mathrm{ml}(90 \mu \mathrm{g} /$ $\mathrm{ml})$ puromycin.

Cell viability assay. Cell viability and half maximal inhibitory concentration $\left(\mathrm{IC}_{50}\right)$ were analyzed using the Cell Counting kit-8 (CCK-8, cat. no. C0037; Beyotime Institute of Biotechnology) assay. Cells were seeded at $2.5 \times 10^{3}$ cells/ well in 96-well plates for $24 \mathrm{~h}$ prior to experimentation, after which various concentrations of DDP $(0-1 \mu \mathrm{g}), 5-\mathrm{FU}$ $(0-500 \mu \mathrm{g})$ or doxorubicin $(0-24 \mu \mathrm{M})$ were added to each well; the CCK-8 assay was performed after $48 \mathrm{~h}$. Briefly, $10 \mu$ l CCK-8 reagent was added to each well and incubated for $2 \mathrm{~h}$ at $37^{\circ} \mathrm{C}$. Absorbance was subsequently measured at $450 \mathrm{~nm}$ using a Synergy 2 Multi-Mode microplate reader (BioTek Instruments, Inc., Winooski, VT, USA). The assay was conducted in quadruplicate for each sample and three parallel experiments were performed. The $\mathrm{IC}_{50}$ values of 5-FU, DDP and doxorubicin were calculated using GraphPad Prism (Version 5.01; GraphPad Software, Inc., La Jolla, CA, USA).

Dual luciferase reporter assay. The dual luciferase reporter assay was conducted according to the manufacturer's protocol (Dual-Luciferase ${ }^{\circledR}$ Reporter Assay system, cat. no. E1910; Promega Corporation, Madison, WI, USA). The luciferase detection kit was used to analyze cells, which were transfected with MDR1 promoter reporter plasmids or blank plasmids. Briefly, $2 \times 10^{5}$ cells were seeded in a 6 -well plate $24 \mathrm{~h}$ prior to 
Table I. Patient clinical parameters.

\begin{tabular}{lr}
\hline Variable & $\mathrm{n}$ \\
\hline Age (years) & \\
$\leq 50$ & 19 \\
$>50$ & 30 \\
Sex & \\
Male & 45 \\
Female & 4 \\
Liver cirrosis & \\
Yes & 13 \\
No & 36 \\
Histological differentiation & \\
High & 5 \\
Moderate & 15 \\
Poor & 29 \\
T stage & \\
I + II & 19 \\
III + IV & 30 \\
Metastasis & \\
Yes & 16 \\
No & 33 \\
\hline
\end{tabular}

experimentation. The MDR1 promoter ranging from -1,040 kbp to +288 bp was cloned by PCR reaction using HepG2 genomic DNA as a template. The PCR products were sequenced and subjected to BLAST analysis (https://blast.ncbi.nlm.nih. gov/Blast.cgi). The pRL-SV40-blank vector (cat. no. 27163; Addgene Inc., Cambridge, MA, USA) was digested with KpnI (cat. no. R0142S; New England Biolabs, Inc., Ipswich, MA, USA) and HindIII (cat. no. R0104S; New England Biolabs, Inc.). PCR products were mixed with digested blank vectors for ligation. The ligation products were then transformed into competent DH5 $\alpha$ bacteria (cat. no. 9057, Takara Biotechnology Co., Ltd., Dalian, China) for cloning and plasmid amplification. Subsequently, $2 \mu \mathrm{g}$ constructed pRL-SV40-MDR1 reporter vector or pRL-SV40-blank vector were mixed with Lipofectamine ${ }^{\circledR} 2000$ (Invitrogen; Thermo Fisher Scientific, Inc.) at room temperature for $30 \mathrm{~min}$, after which the mixture was added to the cells and incubated at $37^{\circ} \mathrm{C}$ for $24 \mathrm{~h}$. Cells were then lysed with lysis buffer after washing with PBS twice, and luciferin substrate was mixed with the lysate for $5 \mathrm{~min}$. The firefly luciferase reporter signal was detected by a microplate spectrophotometer in luminescence format. Renilla luciferase activity was also measured for normalization. Three replicate wells were used for each condition and the experiment was conducted three times for validation.

Intracellular accumulation of doxorubicin. Once the cells reached $70-80 \%$ confluence in 6-well plates, a final concentration of $10 \mu \mathrm{M}$ doxorubicin was added to each well and incubated at $37^{\circ} \mathrm{C}$ for $3 \mathrm{~h}$. Subsequently, the cells were washed and resuspended at $5-10 \times 10^{5}$ cells $/ \mathrm{ml}$ in PBS. After washing with PBS, the cells were observed by fluorescence microscopy and analyzed by flow cytometry: Excitation wavelength,
$488 \mathrm{~nm}$; emission wavelength, $575 \mathrm{~nm}$. For intracellular doxorubicin analysis, red mean fluorescence intensity (MFI) of the gated cells was measured to determine drug accumulation by flow cytometry (EPICS XL-MCL; Beckman Coulter, Inc., Brea, CA, USA) using with ModFit LT software version 2.0 (Beckman Coulter, Inc.). Briefy, $1 \times 10^{4}$ cells were collected under the following settings: forward scatter (FSC), $200 \mathrm{mV}$; side scatter (SSC), $250 \mathrm{mV}$ and fluorescein isothiocyanate (FITC) channel, $400 \mathrm{mV}$. Single cells in the FSC-SSC window were gated for $575 \mathrm{~nm}$ channel signal detection.

Confocal microscopy. Cells were detected by indirect immunofluorescence. Cells were fixed with 1:1 acetone-methanol at room temperature for $30 \mathrm{~min}$, and then incubated with the following primary antibodies overnight at $4^{\circ} \mathrm{C}$, TWIST (1:100), vimentin (1:100) and E-cadherin (1:100). Antigenic sites were subsequently localized using FITC-labeled secondary antibody (E-cadherin; 1:100, cat. no. 111095045; Jackson ImmunoResearch Europe Ltd., Ely, UK) or TRITC-conjugated goat anti-rabbit secondary antibody (Vimentin; 1:100, cat. no. 111025045; Jackson ImmunoResearch Europe Ltd.). DAPI was used to stain the nuclei. Images were captured under a Leica laser scanning confocal microscope (TCS SP5; Leica Microsystems GmbH).

Western blot analysis. Western blotting was performed as previously described (18). Cells were collected and lysed with radioimmunoprecipitation assay lysis buffer $[50 \mathrm{mM}$ Tris- $\mathrm{HCl}$ (pH 8.0), $150 \mathrm{mM}$ sodium chloride, 1.0\% Igepal CA-630 (NP-40), $0.5 \%$ sodium deoxycholate). Protein quantification was measured using NanoDrop 3000 (NanoDrop; Thermo Fisher Scientific, Inc., Wilmington, DE, USA) at A280. Total proteins (60-80 $\mu \mathrm{g}$ ) were separated by 10\% SDS-PAGE and were transferred to polyvinylidene difluoride membranes (pore size, $0.22 \mu \mathrm{M}$ ). After washing twice with Tris-buffered saline containing $0.1 \%$ Tween-20 (TBST), the membranes were incubated with $5 \%$ not-fat dried milk in TBST at room temperature for $1 \mathrm{~h}$, and the membranes were incubated with primary antibodies against TWIST (1:250, cat. no. ab49254; Abcam), vimentin (1:1,000, cat. no. 3932; Cell Signaling Technology Inc.), E-cadherin (1:500, cat. no. 3195; Cell Signaling Technology Inc.), MDR1 (1:1,000, cat. no. ab170904), multidrug resistance associated protein 1 (MRP1, 1:1,000, cat. no. 14685; Cell Signaling Technology Inc.), N-cadherin (1:500, cat. no. 13116; Cell Signaling Technology Inc.) and Snail (1:1,000, cat. no. 9782; Cell Signaling Technology Inc.) and $\beta$-actin (1:2,000, cat. no. 4970; Cell Signaling Technology Inc.) at $4^{\circ} \mathrm{C}$ overnight. After a further two washes in TBST, the membranes were incubated with horseradish peroxidase-conjugated secondary antibodies $(1: 2,000$, cat. no. 111035003; Jackson ImmunoResearch Europe Ltd.) for $1 \mathrm{~h}$ at room temperature. Signals were detected on X-ray film using an enhanced chemiluminescence detection system (Pierce; Thermo Fisher Scientific, Inc.).

Cell migration and wound-healing assays. The migratory ability of cells was evaluated using Transwell inserts with $8 \mathrm{~mm}$ pores (Corning Incorporated, Corning, NY, USA), as previously described (19). Briefly, $2 \times 10^{5}$ cells in $100 \mu 1$ serum-free medium and $600 \mu \mathrm{l}$ RPMI-1640 containing 10\% FBS were added to the 


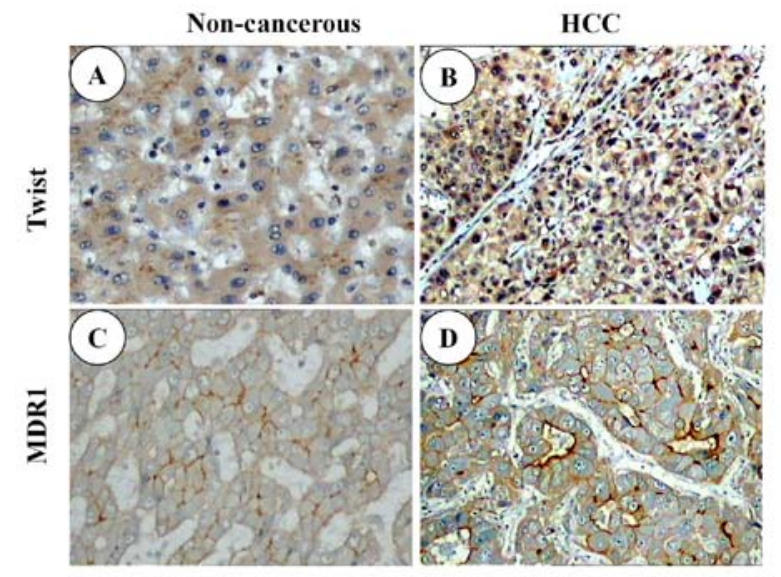

G

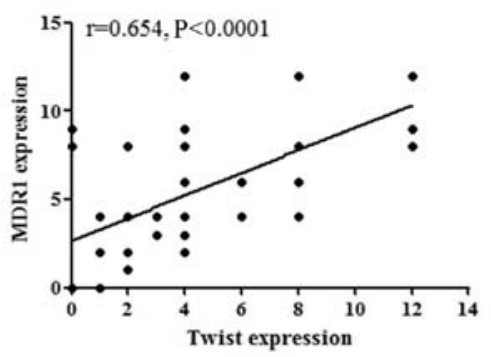

$\mathbf{E}$
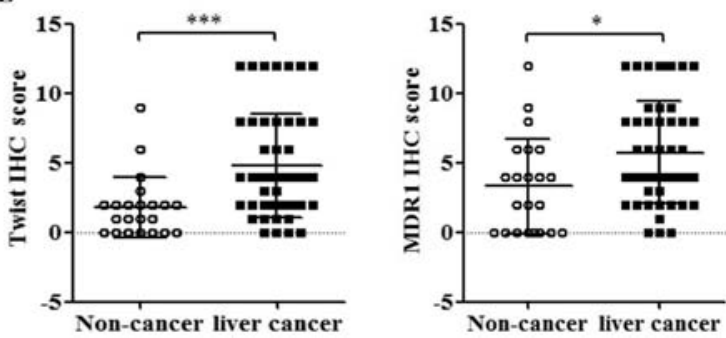

Figure 1. High expression of TWIST is associated with high expression of MDR1 in liver tissues. (A-D) Representative immunohistochemical staining of TWIST and MDR1 in clinical liver cancer and corresponding non-cancerous samples (magnification, x200). (E and F) Immunostaining intensity of TWIST and MDR1 was significantly increased in cancer specimens compared with in non-cancerous tissues. ${ }^{* * * *} \mathrm{P}<0.001,{ }^{*} \mathrm{P}<0.05$. (G) Correlation between TWIST and MDR1 expression in cancer ( $\mathrm{r}=0.654, \mathrm{P}<0.0001$, Pearson's correlation coefficient). TWIST, twist family bHLH transcription factor 1 .

upper and lower chambers, respectively. After 48-h incubation at room temperature, non-migrated cells were removed with a cotton swab, and the membrane inserts were fixed with 1:1 methanol and acetone for $15 \mathrm{~min}$, and stained with $0.1 \%$ crystal violet for $45 \mathrm{~min}$. Migrated cells were counted within five representative fields in triplicate under a microscope (TS100-F serial; Nikon Corporation, Tokyo, Japan).

For the wound-healing assay, normal cells or cells infected with si-TWIST or NC $\left(5 \times 10^{4}\right)$ for $24 \mathrm{~h}$ were harvested and seeded into 6-well plates in RPMI-1640 supplemented with $0.5 \% \mathrm{FBS}$; the cells were then cultured for $24 \mathrm{~h}$, according to a previously published study (20). Once the cells reached $80 \%$ confluence, three parallel scratches were made using a 10- $\mu$ l pipette tip; $1 \mathrm{X}$ PBS was used to remove the free-floating cells and serum-free medium was added to the 6-well plate. After 24, 48 and $72 \mathrm{~h}$, a microscope in bright light mode (BX51; Olympus Corporation, Tokyo, Japan) was used to observe the distance migrated by the cells. Wound-healing area was calculated according to the following equation: Area $=$ length $\mathrm{x}$ width. The wound-healing rate at 24,48 and $72 \mathrm{~h}$ was calculated through normalization to the area at $0 \mathrm{~h}$. Triplicate wells were used for each treatment.

Statistical analysis. SPSS version 16.0 software package (SPSS, Inc., Chicago, IL, USA) and GraphPad Prism were used for statistical analysis and data plotting. The experiments were repeated in triplicate and the data are expressed as the means \pm sttandard deviation. For comparisons between two groups, a Mann-Whitney U test was performed. When multiple comparisons were made, the Bonferroni correction was applied. Pearson's correlation coefficient was used for association analysis. $\mathrm{P}<0.05$ was considered to indicate a statistically significant difference.

\section{Results}

TWIST is highly expressed in cancer specimens and is correlated with MDRl. To evaluate the expression of TWIST in liver cancer, IHC was used to examine TWIST expression in 49 specimens and 22 corresponding non-cancerous specimens. TWIST was predominantly expressed in the nuclei, and mixed nuclear and cytoplasmic expression was also observed in some tumor cells (Fig. 1A and B). In addition, MDR1 was predominantly expressed in epithelial liver tumor cell membranes, and cytoplasmic expression was also observed in some tumor cells (Fig. 1C and D). High expression of TWIST was detected in 17/49 cancer samples and 2/22 non-cancerous tissues ( $\mathrm{P}=0.0004$; Fig. 1E). High expression of MDR1 was detected in 23/49 cancer samples and 6/22 non-cancerous tissues $(\mathrm{P}=0.01$; Fig. $1 \mathrm{~F})$. The present study also demonstrated that there was a positive correlation between TWIST and MDR1 expression (Fig. 1G; Pearson's correlation coefficient, $r=0.654, \mathrm{P}<0.0001)$.

Association between MDRI and EMT markers in cancer samples. The present study aimed to analyze the effects of MDR1 on liver cancer cell EMT transformation (Fig. 2). The association between MDR1, and membrane E-cadherin and vimentin expression in cancer samples was analyzed using Pearson's correlation coefficient. A negative correlation was determined between MDR1 and membrane E-cadherin expression ( $\mathrm{r}=-0.407, \mathrm{P}=0.004$; Fig. $2 \mathrm{~B}$ and $\mathrm{D}$ ); however, there 


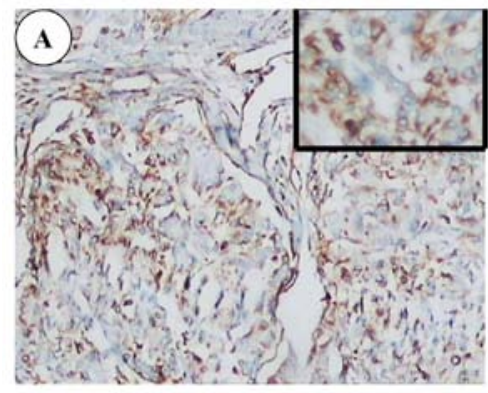

Vimentin

C

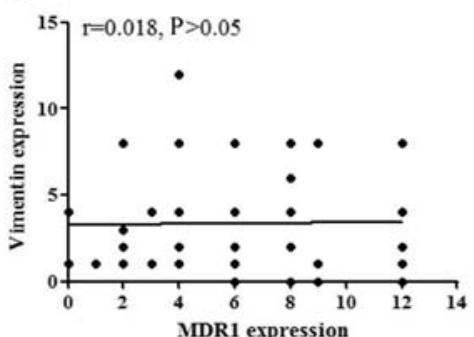

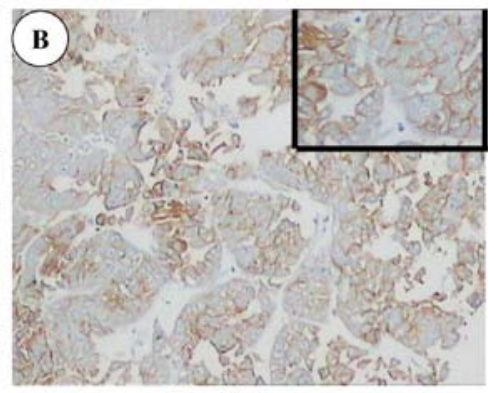

E-cadherin

D

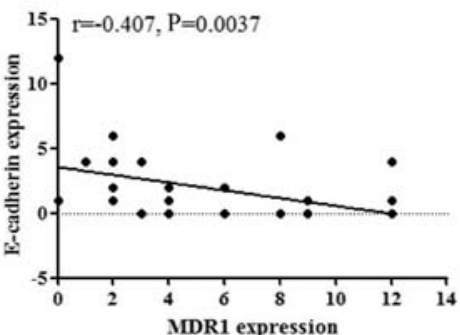

Figure 2. Association between MDR1 and epithelial-mesenchymal transition markers in liver cancer samples. (A and B) Representative immunohistochemical staining of vimentin and E-cadherin in cancer tissues (magnification, $x 200$; magnification of inset images, $\mathrm{x} 400$ ). (C) Correlation between vimentin and MDR1 expression in cancer $(r=0.018, P>0.05$, Pearson's correlation coefficient). (D) Correlation between E-cadherin and MDR1 expression in cancer ( $\mathrm{r}=-0.407$, $\mathrm{P}=0.004$, Pearson's correlation coefficient).

A

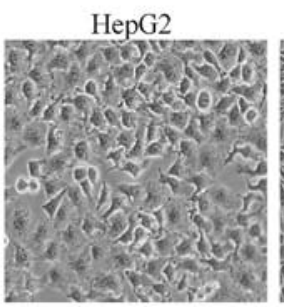

\section{R-HepG2}

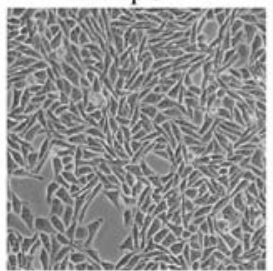

B

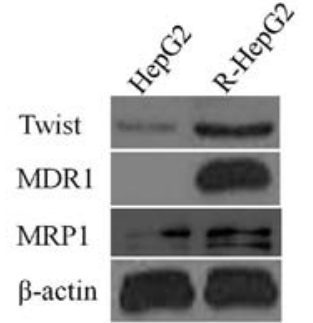

C

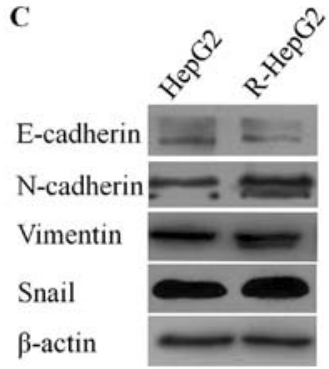

D
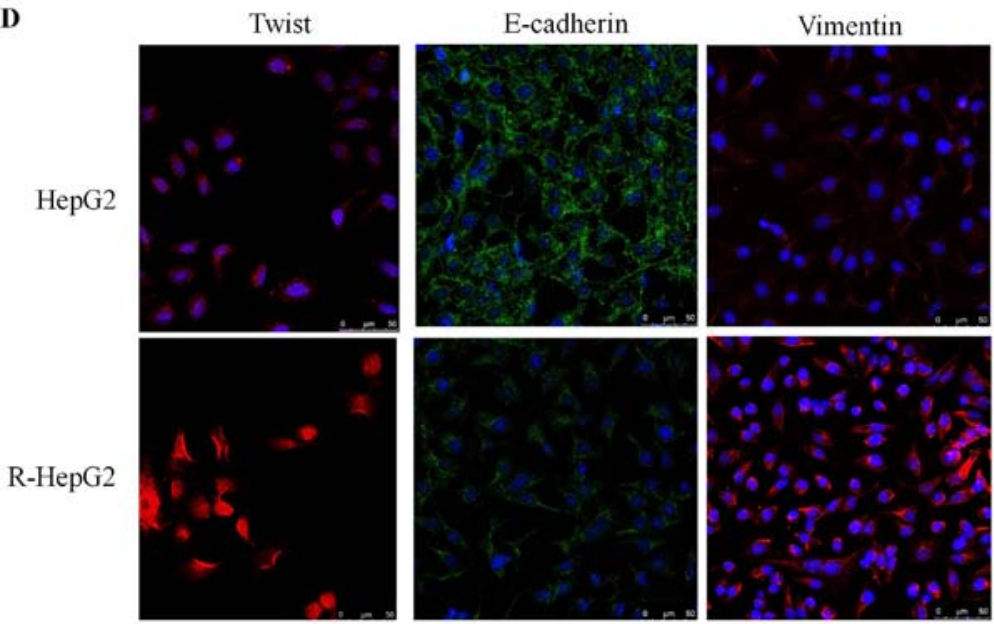

Figure 3. R-HepG2 cells acquire an EMT phenotype. (A) Cell morphology was observed under a microscope (magnification, x400). Expression levels of EMT marker proteins were detected by (B and C) western blotting (D) and immunofluorescence staining (scale bars, $50 \mu \mathrm{m}$ ). EMT, epithelial-mesenchymal transition; MRP1, multidrug resistance associated protein 1; R-HepG2, doxorubicin-resistance HepG2; TWIST, twist family bHLH transcription factor 1.

was no correlation between MDR1 and vimentin ( $\mathrm{r}=0.018$, $\mathrm{P}>0.05$; Fig. $2 \mathrm{~A}$ and $\mathrm{C}$ ). These results indicated that MDR1 expression may be associated with EMT.
R-HepG2 cells exhibit EMT characteristics. To determine whether R-HepG2 cells could obtain EMT characteristics, R-HepG2 cells were cultured and analyzed. As expected, 
A

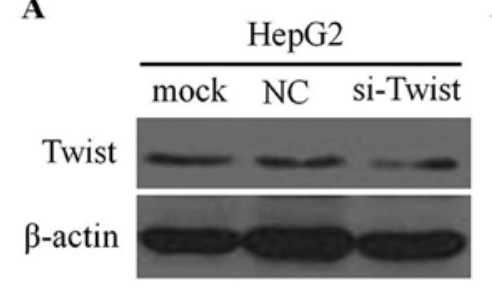

C

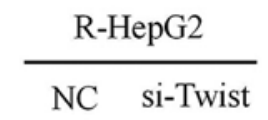

MDR1

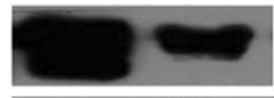

MRP1

$\beta$-actin
B

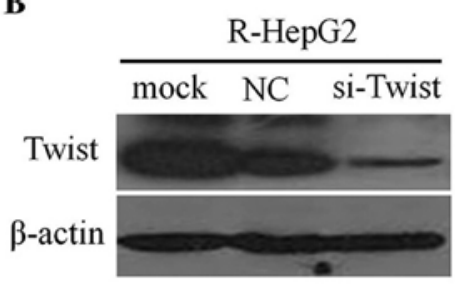

D

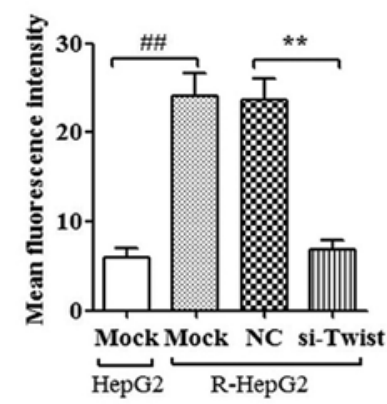

Figure 4. Knockdown of TWIST expression suppresses MDR1 promoter activity and MDR1 protein level in R-HepG2 cells. (A and B) Protein expression levels of TWIST were not markedly affected in HepG2 and NC cells, but were markedly decreased in R-HepG2 cells in response to si-TWIST. (C) MDR1 expression was markedly decreased in R-HepG2 si-TWIST-infected cells. (D) MDR1 promoter firefly luciferase activity was measured and normalized against Renilla luciferase activity. ${ }^{\# \#} \mathrm{P}<0.01$ compared with HepG2, ${ }^{* *} \mathrm{P}<0.01$ compared with NC. MDR1, multidrug resistance protein 1 ; MRP1, multidrug resistance associated protein 1; NC, negative control; R-HepG2, doxorubicin-resistance HepG2; si-TWIST, TWIST-specific shRNA; TWIST, twist family bHLH transcription factor 1 .

R-HepG2 cells exhibited phenotypic alterations consistent with EMT. R-HepG2 cells possessed elongated, irregular fibroblastic morphology compared with HepG2 parental cells, which exhibited an irregular flat morphology and few pseudopodia (Fig. 3A). Notably, the expression levels of the epithelial molecule E-cadherin were decreased and the expression levels of mesenchymal markers, including $\mathrm{N}$-cadherin, vimentin, TWIST and Snail were increased in R-HepG2 cells, as determined by immunofluorescence staining and western blotting (Fig. 3B-D). These findings suggested that R-HepG2 cells obtained specific EMT molecular markers.

Knockdown of TWIST decreases MDRI expression in $R$-HepG2 cells. To determine the knockdown efficiency of TWIST-specific shRNA-containing lentiviruses, si-TWIST and NC cells were harvested (Fig. 4A and B). The data demonstrated that the protein expression levels of TWIST were reduced in R-HepG2 si-TWIST cells compared with in mock or NC cells (Fig. 4B); however, no marked effect was detected in HepG2 cells (Fig. 4A). Furthermore, knockdown of TWIST decreased MDR1 protein expression in R-HepG2 cells; however, it had no effect on MRP1 protein expression (Fig. 4C). In addition, luciferase assays indicated that knockdown of TWIST significantly suppressed MDR1 promoter activity in R-HepG2 cells (Fig. 4D). These results supported the possibility that knockdown of TWIST may suppress MDR1 expression in cancer cells.

Knockdown of TWIST reduces drug efflux from $R$-Hep $G 2$ cells. To evaluate the effects of TWIST on regulating MDR1 transporter activity, R-HepG2 cells were harvested and treated with doxorubicin, which emits a natural red fluorescence.
Intracellular doxorubicin fluorescence intensity was measured by fluorescence microscopy. Red fluorescence was stronger in si-TWIST cells compared with in NC cells following treatment with doxorubicin, indicating increased doxorubicin accumulation in si-TWIST cells (Fig. 5A). Flow cytometry also revealed that the intracellular concentration of doxorubicin was augmented in si-TWIST cells (Fig. 5B and C). The red MFI was significantly increased in si-TWIST cells compared with in NC cells treated with doxorubicin for $3 \mathrm{~h}$. Taken together, these data suggested that knockdown of TWIST may decrease drug efflux, leading to higher intracellular accumulation of doxorubicin in R-HepG2 cells.

Knockdown of TWIST increases the cytotoxicity of chemotherapeutic drugs. Knockdown of TWIST suppressed MDR1, thus indicating that altering TWIST may increase drug sensitivity in cancer cells. To assess this hypothesis, the cytotoxicity index of 5-FU, DDP and doxorubicin was determined in si-TWIST cells (Fig. 6). The results demonstrated that TWIST knockdown in R-HepG2 cells resulted in 2-10-fold increased cytotoxicity of the chemotherapeutic drugs (5-FU, DDP and doxorubicin) compared with in NC cells, as determined by CCK- 8 assays $(\mathrm{P}<0.05$, $\mathrm{U}$ test; Fig. 6D-F). The $\mathrm{P}$-value provided refers to the comparison of the end-point dosage between the two groups. The findings were further confirmed by the $\mathrm{IC}_{50}$ assay; si-TWIST resulted in decreasing 5-FU, DDP and doxorubicin $\mathrm{IC}_{50}$ values. These data indicated that knockdown of TWIST may lead to enhanced sensitivity of R-HepG2 cells to anticancer drugs.

Knockdown of TWIST expression inhibits the migratory ability of R-HepG2 cells. The wound-healing assay revealed 
A

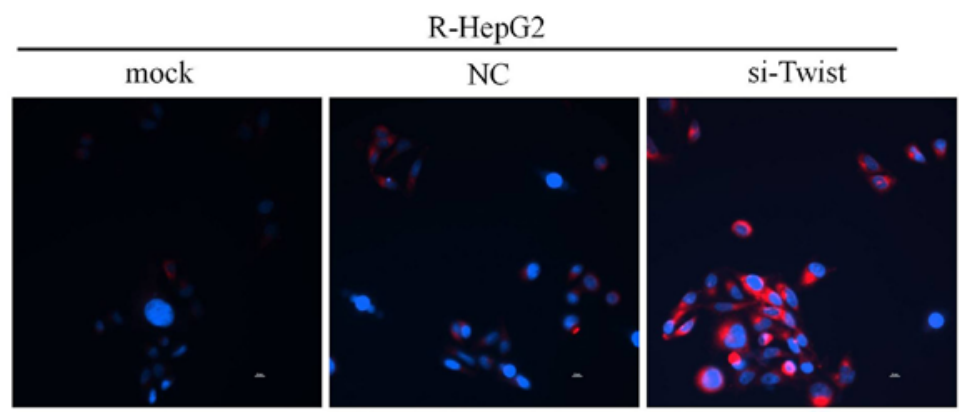

B
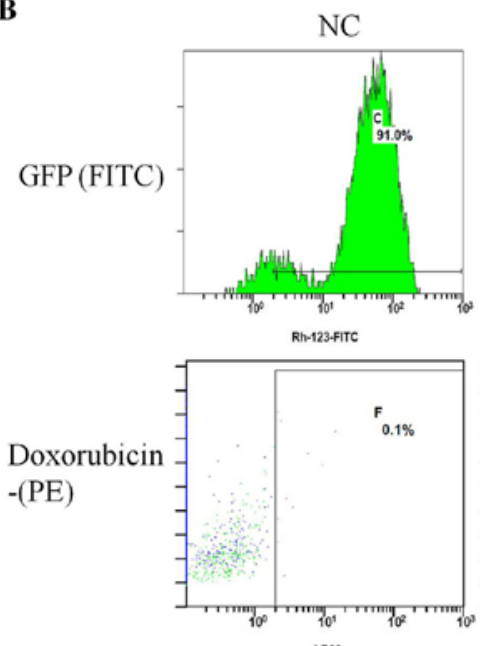

si-Twist

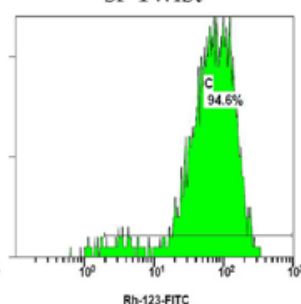

Rh-123.FiT

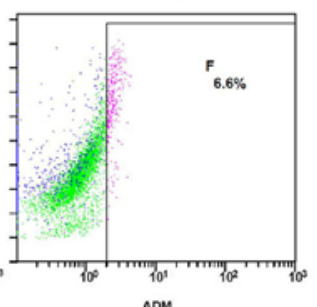

C

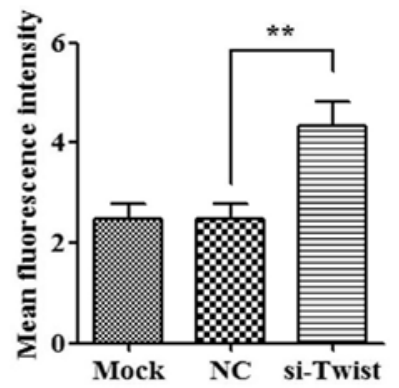

Figure 5. Knockdown of TWIST increases intracellular accumulation of doxorubicin in R-HepG2 cells. (A) Fluorescence intensity of intracellular doxorubicin was recorded by fluorescence microscopy (magnification, x400). (B and C) Intracellular accumulation of doxorubicin was analyzed by flow cytometry to determine the effects of si-TWIST on the accumulation of doxorubicin in R-HepG2 cells. ${ }^{* *} \mathrm{P}<0.01$ compared with NC. FITC, fluorescein isothiocyanate; GFP, green fluorescent protein; NC, negative control; PE, phycoerythrin; R-HepG2, doxorubicin-resistance HepG2; si-TWIST, TWIST-specific shRNA; TWIST, twist family bHLH transcription factor 1.

A

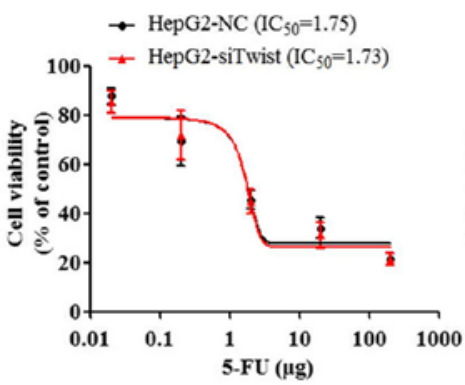

D

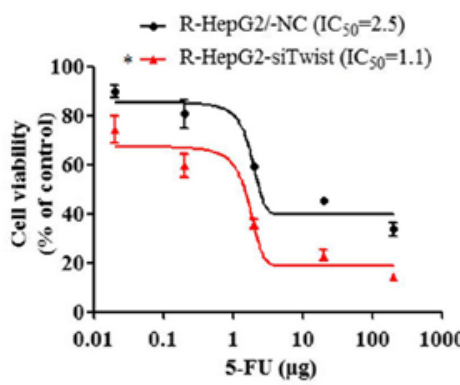

B

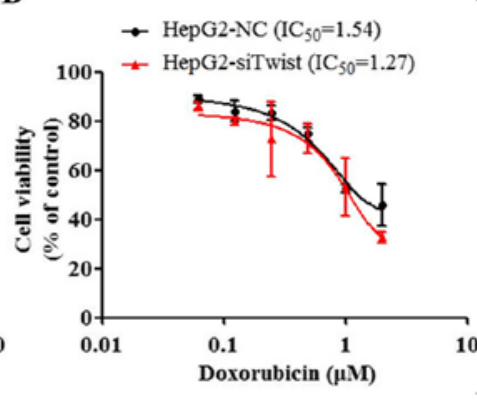

$\mathbf{E}$

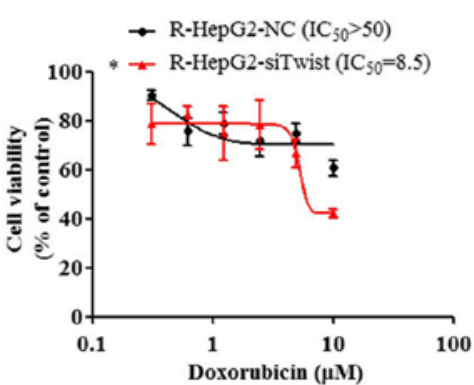

C

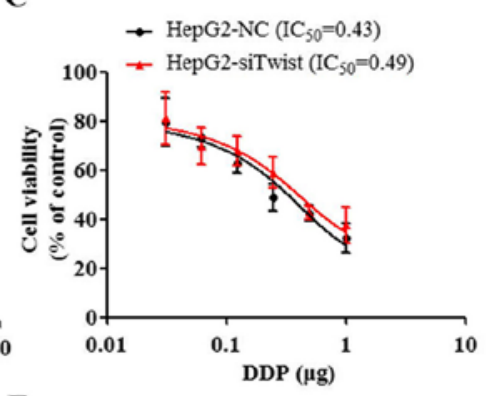

F

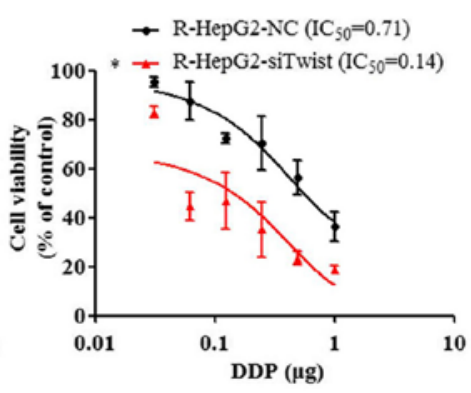

Figure 6. Knockdown of TWIST enhances the sensitivity of R-HepG2 cells to the cytotoxic effects of 5-FU, DDP and doxorubicin. (A-F) Cytotoxicity and IC 50 in HepG2 and R-HepG2 were measured. Data are presented as the means \pm standard deviation (n=3). (D) Treatment with $200 \mu \mathrm{g} / \mathrm{ml} 5$-FU exhibited higher cytotoxicity in si-TWIST cells than NC cells; " $\mathrm{P}<0.05$ compared with NC. (E) Treatment with $10 \mu \mathrm{M}$ doxorubicin exhibited higher cytotoxicity in si-TWIST cells than NC cells; ${ }^{*} \mathrm{P}<0.05$ compared with NC. (F) Treatment with $1 \mu \mathrm{g} / \mathrm{ml}$ DDP exhibited higher cytotoxicity in si-TWIST cells than NC cells; "P<0.05 compared with NC. 5-FU, 5-fluorouracil; DDP, cisplatin; NC, negative control; R-HepG2, doxorubicin-resistance HepG2; si-TWIST, TWIST-specific shRNA; TWIST, twist family bHLH transcription factor 1 . 

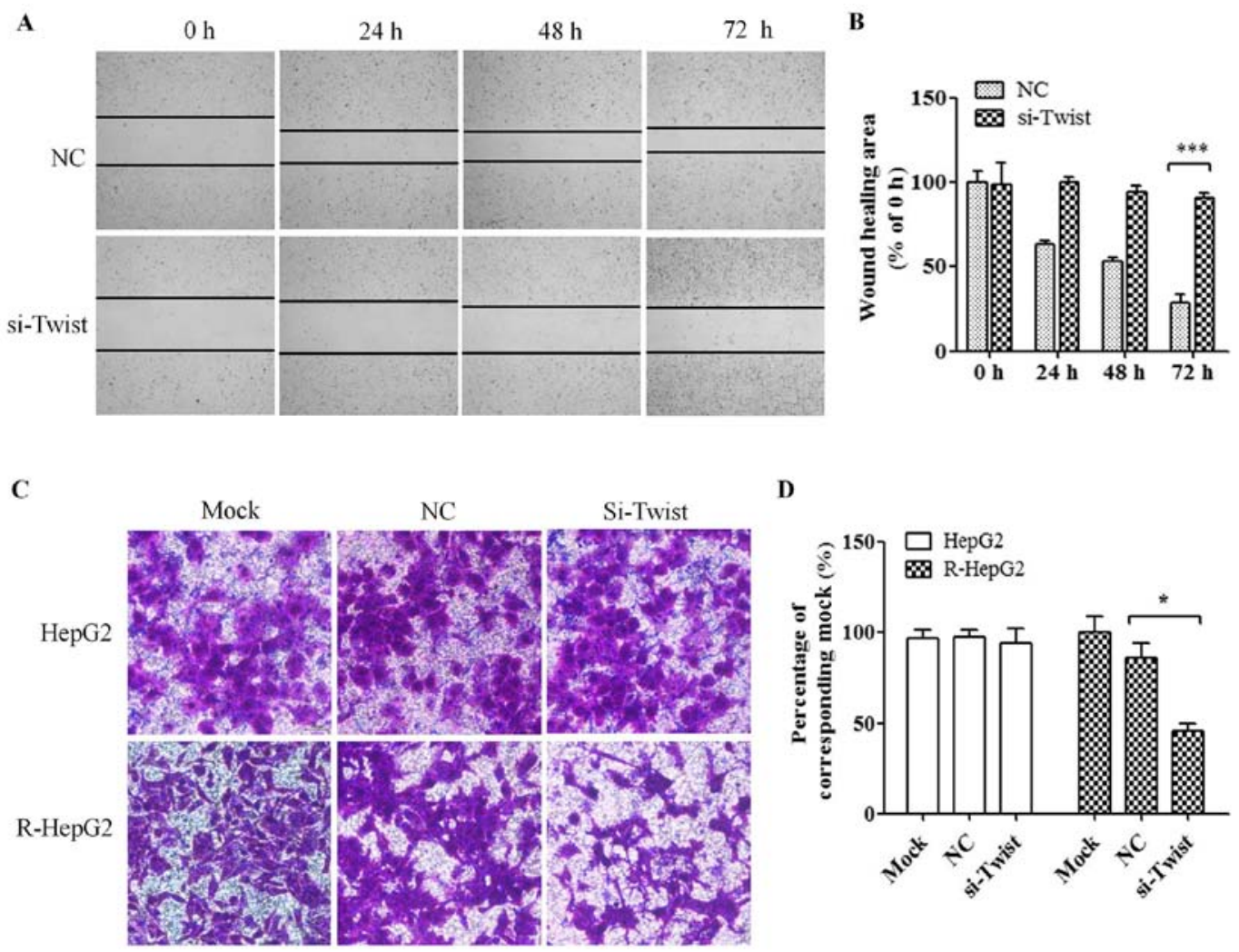

D

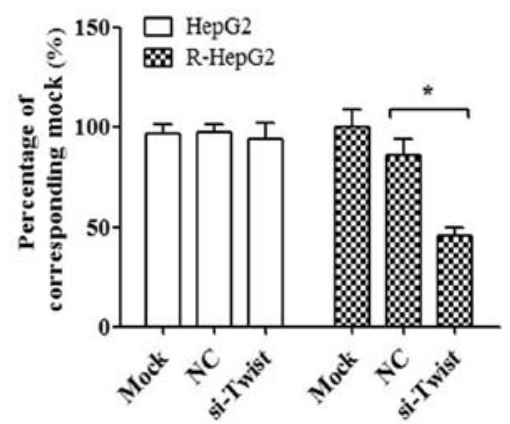

Figure 7. Knockdown of TWIST inhibits the migration of R-HepG2 cells. Cell motility was analyzed by wound-healing and Transwell migration assays. (A and B) Representative images of the wound-healing assay and quantification via the wound-healing area rate method (magnification, $\mathrm{x} 100) .{ }^{* * *} \mathrm{P}<0.001$, si-TWIST vs. NC group at $72 \mathrm{~h}$. (C and D) Following the Transwell migration assay, migrated cells were stained with $0.1 \%$ crystal violet (magnification, $\mathrm{x} 200$ ). "P<0.05. NC, negative control; R-HepG2, doxorubicin-resistance HepG2; si-TWIST, TWIST-specific shRNA; TWIST, twist family bHLH transcription factor 1.

A

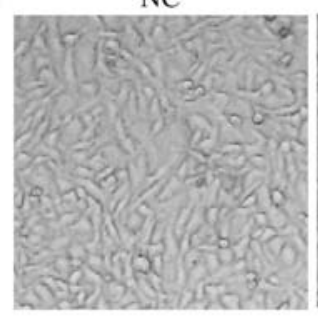

C
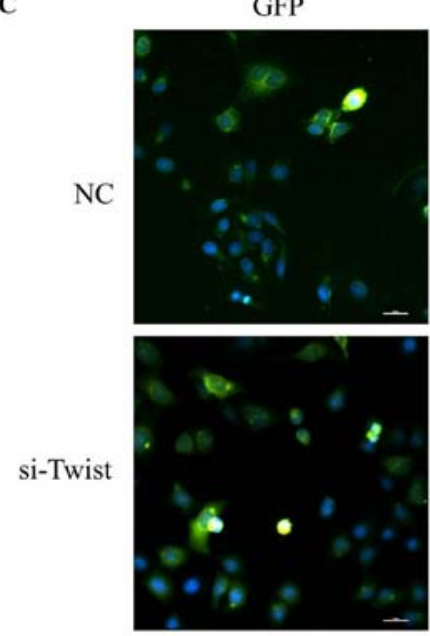

si-Twist

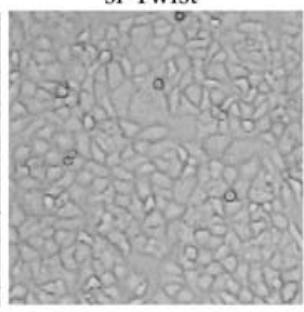

E-cadherin
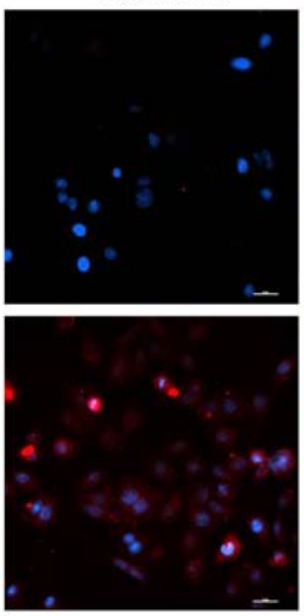

B

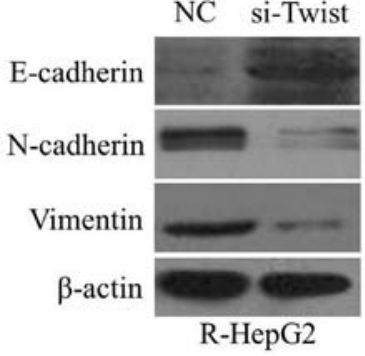

Vimentin
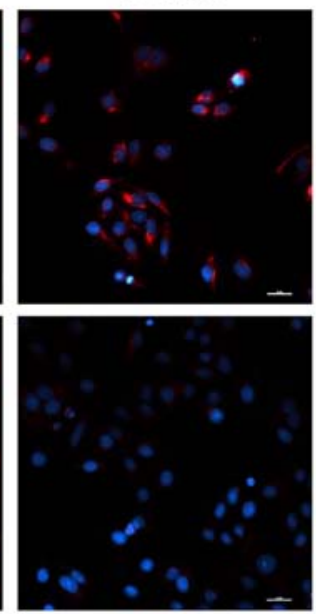

Figure 8. Knockdown of TWIST reverses EMT to mesenchymal-epithelial transition in R-HepG2 cells. (A) Images of cell morphology were captured (magnification, x200). (B) EMT marker proteins were detected by (B) western blotting and (C) immunofluorescence staining (scale bars, $50 \mu \mathrm{m})$. EMT, epithelial-mesenchymal transition; NC, negative control; R-HepG2, doxorubicin-resistance HepG2; si-TWIST, TWIST-specific shRNA; TWIST, twist family bHLH transcription factor 1 . 
that knockdown of TWIST suppressed cell migration in R-HepG2 cells (U test; Fig. 7A and B). Transwell assay was used to detect the effects of TWIST silencing on R-HepG2 cell motility. The migration of si-TWIST cells was significantly decreased compared with mock and NC cells (U test; Fig. 7C and D); these findings were similar to the findings of the wound-healing assay.

Knockdown of TWIST reverses EMT to mesenchymal-epithelial transition (MET) in R-HepG2 cells. R-HepG2-si-TWIST cells exhibited round cell-like phenotype (Fig. 8A). Furthermore, the expression levels of E-cadherin were elevated, whereas vimentin and $\mathrm{N}$-cadherin were reduced in R-HepG2-si TWIST cells (Fig. 8B and C). These findings indicated that downregulation of TWIST may reverse the EMT phenotype to a MET phenotype.

\section{Discussion}

TWIST has been reported to promote EMT resulting in the promotion of tumor invasion $(21,22)$. TWIST expression is elevated in hepatocellular carcinoma, breast cancer, nasopharyngeal carcinoma and oral carcinoma (21,23-25). Previous studies have revealed a novel function of TWIST, and it has been reported to be involved in the development of acquired chemoresistance in human cancer cells $(15,16,26)$. In the present study, TWIST and MDR1 were expressed to a significantly higher level in liver tissues compared with in non-cancerous tissues. In addition, the present study demonstrated that there was a positive correlation between TWIST and MDR1 expression in liver cancer tissues.

Accumulating evidence has demonstrated that MDR cancer cells are associated with the EMT process. For example, tamoxifen-resistant MCF7/TR breast cancer cells acquire EMT features (27). Similarly, cisplatin-resistant cervical cancer cells possess more EMT characteristics, and exhibit increased migratory abilities and invasiveness (28). Furthermore, gemcitabine-resistant pancreatic cancer cells possess EMT characteristics (29). The present study revealed that increased MDR1 and MRP1 expression was detected in R-HepG2 cells alongside acquired EMT phenotypic characteristics, reduced E-cadherin, and elevated vimentin, Snail and TWIST expression. Due to the lack of a suitable hepatocellular carcinoma-derived drug-resistant cell line, HepG2 cells were selected for use in this study. Although HepG2 cells have been identified as a hepatoblastoma cell line (17), the evaluation of its role in drug-induced resistance is still meaningful, as drug resistance is a prevalent phenomenon in various types of cancer. Therefore, parental HepG2 and drug-resistant R-HepG2 cells were used in the present study to evaluate liver cancer drug resistance. HepG2 and drug-resistant R-HepG2 cells have been studied in numerous papers on drug resistance (30-32). In addition, hepatoblastoma samples are difficult to collect, as they are rare in our hospital. For the present study, both hepatocellular carcinoma and hepatoblastoma cases were therefore collected. The results provided evidence to suggest that there was a negative correlation between MDR1 and E-cadherin expression. The results indicated that a link may exist between MDR and EMT in cancer development. Whether the expression of MDR1 is regulated by TWIST remains to be elucidated.
Overexpression of MDR1 causes the efflux of various hydrophobic compounds and xenobiotics, resulting in drug resistance. The transcriptional levels of MDR1 are regulated by numerous pathways, such as those mediated by activator protein 1, nuclear transcription factor Y subunit $\alpha$ (NF-Y), GC rich-box and p53, and even methylation and acetylation (33). In addition, MDR1 is regulated by microRNAs (miRs), such as miR-495 and miR-127 (34,35). Ma et al (36) revealed that zinc fingers and homeoboxes 2 decreases NF-Y-mediated activation of MDR1 transcription and improves the effects of chemotherapy in hepatocellular carcinoma cells. A potential transcriptional regulatory role of TWIST1 has been confirmed in MDR. Zhu et al (26) reported that knockdown of TWIST decreases MDR1 expression in Hela cells, inhibits cell proliferation, suppresses Rhodamine 123 efflux activity of cells and enhances cytotoxicity following treatment with DDP. In the present study, knockdown of TWIST suppressed MDR1 expression and significantly decreased MDR1 promoter activity. Furthermore, knockdown of TWIST reduced drug efflux, leading to increased intracellular doxorubicin levels in R-HepG2 cells and increased sensitivity of R-HepG2 cells to these chemotherapeutic drugs. These findings indicated that knockdown of TWIST may enhance the cytotoxicity of chemotherapeutic drugs in R-HepG2 cells by inhibiting MDR1.

It has previously been demonstrated that EMT inducers can increase migratory, invasive potential and promote MDR by upregulating ABC transporters, which efflux chemotherapeutic drugs (37). EMT regulators, such as TWIST, Snail and Zeb1 transcription factors, and some signal pathways, including transforming growth factor $\beta$, Wnt and Notch, are known to induce EMT (38). In agreement with those previous studies, the present results demonstrated that knockdown of TWIST inhibited the migratory ability of R-HepG2 cells and reversed EMT to MET in R-HepG2 cells. In addition, the involvement of other EMT inducers in TWIST/MDR1-mediated R-HepG2 cells will be investigated in future studies.

\section{Acknowledgements}

The authors would like to thank Dr Kangrong Cai (Department of Analytic Center, Guangdong Medical University) for setting up the flow cytometer and providing software instruction.

\section{Funding}

The present study was supported by the National Natural Science Foundation of China (grant nos. 81441118 and 81572782), the Project of Constructing Strong Province of Traditional Chinese Medicine by the Traditional Chinese Medicine Bureau of Guangdong Province, China (grant no. 20152150), the Sci-Tech Project Foundation of Zhanjiang City, China (grant nos. 2013C301015 and 2015A01040), and the Key Cultivation Project of Guangdong Medical University, China (grant no. Z2015001).

\section{Availability of data and materials}

All data generated or analyzed during this study are included in this published article. 


\section{Authors' contributions}

RL and CW designed the study, conducted experiments and performed data analysis; they also wrote the manuscript. HL performed immunohistochemistry staining, and wrote this part of the Materials and methods and Results. YZ carried out western blotting, and wrote the Results section of the manuscript. CL performed the cell cytotoxicity assay experiment. $\mathrm{XZ}$ and $\mathrm{CY}$ designed the MDR1 gene promoter reporter assay vector, performed the luciferase reporter experiment, revised the manuscript and examined the validity of all experiments. CY also gave final approval of the version to be submitted and published.

\section{Ethics approval and consent to participate}

The present study was approved by the Institutional Ethics Committee of Guangdong Medical University (Zhanjiang, China). All patients provided written informed consent.

\section{Patient consent for publication}

Not applicable.

\section{Competing interests}

The authors declare that they have no competing interests.

\section{References}

1. Wada Y, Takami Y, Tateishi M, Ryu T, Mikagi K and Saitsu H: Impact of more detailed categorization of shrinkage or progression ratio at initial imaging response after sorafenib treatment in advanced hepatocellular carcinoma patients. OncoTargets Ther 8: 3193-3202, 2015.

2. Zender L, Villanueva A, Tovar V, Sia D, Chiang DY and Llovet JM: Cancer gene discovery in hepatocellular carcinoma. J Hepatol 52: 921-929, 2010.

3. Bhangoo MS, Karnani DR, Hein PN, Giap H, Knowles H, Issa C, Steuterman S, Pockros P and Frenette C: Radioembolization with Yttrium-90 microspheres for patients with unresectable hepatocellular carcinoma. J Gastrointest Oncol 6: 469-478, 2015.

4. Song MJ and Bae SH: Newer treatments for advanced hepatocellular carcinoma. Korean J Intern Med (Korean Assoc Intern Med) 29: 149-155, 2014.

5. Follit CA, Brewer FK, Wise JG and Vogel PD: In silico identified targeted inhibitors of P-glycoprotein overcome multidrug resistance in human cancer cells in culture. Pharmacol Res Perspect 3: e00170, 2015.

6. McCormick JW, Vogel PD and Wise JG: Multiple drug transport pathways through human P-glycoprotein. Biochemistry 54: 4374-4390, 2015.

7. Janigro D, Perju C, Fazio V, Hallene K, Dini G, Agarwal MK and Cucullo L: Alternating current electrical stimulation enhanced chemotherapy: A novel strategy to bypass multidrug resistance in tumor cells. BMC Cancer 6: 72, 2006.

8. Rhim AD,MirekET,AielloNM,Maitra A,Bailey JM,McAllisterF, Reichert M, Beatty GL, Rustgi AK, Vonderheide RH, et al: EMT and dissemination precede pancreatic tumor formation. Cell 148 349-361, 2012.

9. Vesuna F, Bergman Y and Raman V: Genomic pathways modulated by Twist in breast cancer. BMC Cancer 17: 52, 2017.

10. Tang $\mathrm{H}$, Massi D, Hemmings BA, Mandalà M, Hu Z, Wicki A and Xue G: AKT-ions with a TWIST between EMT and MET. Oncotarget 7: 62767-62777, 2016.

11. Zhang H, Gong J, Kong D and Liu HY: Anti-proliferation effects of Twist gene silencing in gastric cancer SGC7901 cells. World J Gastroenterol 21: 2926-2936, 2015.
12. Nantajit D, Lin D and Li JJ: The network of epithelial-mesenchymal transition: Potential new targets for tumor resistance. J Cancer Res Clin Oncol 141: 1697-1713, 2015.

13. Jiang ZS, Sun YZ, Wang SM and Ruan JS: Epithelialmesenchymal transition: Potential regulator of $\mathrm{ABC}$ transporters in tumor progression. J Cancer 8: 2319-2327, 2017.

14. Deng JJ, Zhang W, Xu XM, Zhang F, Tao WP, Ye JJ and Ge W: Twist mediates an aggressive phenotype in human colorectal cancer cells. Int J Oncol 48: 1117-1124, 2016.

15. Liu YR, Liang L, Zhao JM, Zhang Y, Zhang M, Zhong WL, Zhang Q, Wei JJ, Li M, Yuan J, et al: Twist1 confers multidrug resistance in colon cancer through upregulation of ATP-binding cassette transporters. Oncotarget 8: 52901-52912, 2017.

16. Lu S, Yu L, Mu Y, Ma J, Tian J, Xu W and Wang H: Role and mechanism of Twist1 in modulating the chemosensitivity of FaDu cells. Mol Med Rep 10: 53-60, 2014.

17. López-Terrada D, Cheung SW, Finegold MJ and Knowles BB: Hep G2 is a hepatoblastoma-derived cell line. Hum Pathol 40: 1512-1515, 2009.

18. Zhang X, Wu C, Xiong W, Chen C, Li R and Zhou G: Knockdown of p54nrb inhibits migration, invasion and TNF- $\alpha$ release of human acute monocytic leukemia THP1 cells. Oncol Rep 35: 3742-3748, 2016.

19. Li R, Zhao Y, Chen J, Shao S and Zhang X: Fisetin inhibits migration, invasion and epithelial-mesenchymal transition of LMP1-positive nasopharyngeal carcinoma cells. Mol Med Rep 9: 413-418, 2014.

20. de Jong E, Winkel P, Poelstra K and Prakash J: Anticancer effects of 15d-prostaglandin-J2 in wild-type and doxorubicin-resistant ovarian cancer cells: Novel actions on SIRT1 and HDAC. PLoS One 6: e25192, 2011.

21. Zhuo X, Chang A, Huang C, Yang L, Xiang Z and Zhou Y: Expression of TWIST, an inducer of epithelial-mesenchymal transition, in nasopharyngeal carcinoma and its clinical significance. Int J Clin Exp Pathol 7: 8862-8868, 2014.

22. Zhang YQ, Wei XL, Liang YK, Chen WL, Zhang F, Bai JW, Qiu SQ, Du CW, Huang WH and Zhang GJ: Over-expressed Twist associates with markers of epithelial mesenchymal transition and predicts poor prognosis in breast cancers via ERK and Akt activation. PLoS One 10: e0135851, 2015.

23. Chun HW and Hong R: Significance of the hedgehog pathway-associated proteins Gli-1 and Gli-2 and the epithelialmesenchymal transition-associated proteins Twist and E-cadherin in hepatocellular carcinoma. Oncol Lett 12: 1753-1762, 2016.

24. Yang L, Hou Y, Yuan J, Tang S, Zhang H, Zhu Q, Du YE, Zhou M, Wen S, Xu L, et al: Twist promotes reprogramming of glucose metabolism in breast cancer cells through PI3K/ AKT and p53 signaling pathways. Oncotarget 6: 25755-25769, 2015.

25. Zhou Y, Zhang H, Zhuo X, Liu Y, Zhang G and Tan Y: Overexpression of TWIST, an epithelial-mesenchymal transition inducer, predicts poor survival in patients with oral carcinoma. Int J Clin Exp Med 8: 9239-9247, 2015.

26. Zhu K, Chen L, Han X, Wang J and Wang J: Short hairpin RNA targeting Twist1 suppresses cell proliferation and improves chemosensitivity to cisplatin in HeLa human cervical cancer cells. Oncol Rep 27: 1027-1034, 2012.

27. Shi XP, Miao S, Wu Y, Zhang W, Zhang XF, Ma HZ, Xin HL, Feng J, Wen AD and Li Y: Resveratrol sensitizes tamoxifen in antiestrogen-resistant breast cancer cells with epithelial-mesenchymal transition features. Int J Mol Sci 14: $15655-15668,2013$.

28. Song J and Li Y: miR-25-3p reverses epithelial-mesenchymal transition via targeting Sema4C in cisplatin-resistance cervical cancer cells. Cancer Sci 108: 23-31, 2017.

29. Wang R, Li Y, Hou Y, Yang Q, Chen S, Wang X, Wang Z, Yang Y, Chen C, Wang Z, et al: The PDGF-D/miR-106a/Twist1 pathway orchestrates epithelial-mesenchymal transition in gemcitabine resistance hepatoma cells. Oncotarget 6: 7000$7010,2015$.

30. Ye CG, Yeung JH, Huang GL, Cui P, Wang J, Zou Y, Zhang $\approx X N$, $\mathrm{He} \mathrm{ZW}$ and Cho $\mathrm{CH}$ : Increased glutathione and mitogenactivated protein kinase phosphorylation are involved in the induction of doxorubicin resistance in hepatocellular carcinoma cells. Hepatol Res 43: 289-299, 2013.

31. Ye CG, Wu WK, Yeung JH, Li HT, Li ZJ, Wong CC, Ren SX, Zhang L, Fung KP and Cho CH: Indomethacin and SC236 enhance the cytotoxicity of doxorubicin in human hepatocellular carcinoma cells via inhibiting P-glycoprotein and MRP1 expression. Cancer Lett 304: 90-96, 2011. 
32. Sun J, Yeung CA, Co NN, Tsang TY, Yau E, Luo K, Wu P, Wa JC, Fung KP, Kwok TT, et al: Clitocine reversal of P-glycoprotein associated multi-drug resistance through down-regulation of

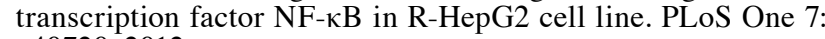
e40720,2012.

33. Jin S and Scotto KW: Transcriptional regulation of the MDR1 gene by histone acetyltransferase and deacetylase is mediated by NF-Y. Mol Cell Biol 18: 4377-4384, 1998.

34. Zou Z, Zou R, Zong D, Shi Y, Chen J, Huang J, Zhu J, Chen L, Bao X, Liu Y, et al: miR-495 sensitizes MDR cancer cells to the combination of doxorubicin and taxol by inhibiting MDR1 expression. J Cell Mol Med 21: 1929-1943, 2017.

35. Feng R and Dong L: Knockdown of microRNA-127 reverses adriamycin resistance via cell cycle arrest and apoptosis sensitization in adriamycin-resistant human glioma cells. Int J Clin Exp Pathol 8: 6107-6116, 2015.
36. Ma H, Yue X, Gao L, Liang X, Yan W, Zhang Z, Shan H, Zhang H, Spear BT and Ma C: ZHX2 enhances the cytotoxicity of chemotherapeutic drugs in liver tumor cells by repressing MDR1 via interfering with NF-YA. Oncotarget 6: 1049-1063, 2015.

37. Saxena M, Stephens MA, Pathak $H$ and Rangarajan A: Transcription factors that mediate epithelial-mesenchymal transition lead to multidrug resistance by upregulating $\mathrm{ABC}$ transporters. Cell Death Dis 2: e179, 2011.

38. Chao HM, Huang HX, Chang PH, Tseng KC, Miyajima A and Chern E: Y-box binding protein-1 promotes hepatocellular carcinoma-initiating cell progression and tumorigenesis via Wnt/ $\beta$-catenin pathway. Oncotarget 8: 2604-2616, 2017. 